\title{
63. NOTA SOBRE GENISTA LOBELII SENSU WILLKOMM EN LA PENÍNSULA IBÉRICA
}

\author{
Salvador TALAVERA, Francisco Javier SALGUEIRO, Llorenç SÁEZ y Baltasar CABEZUDO \\ A note on Genista lobelli sensu Willkomm in the Iberian Peninsula \\ Palabras clave. Genista, Leguminosae, nuevas combinaciones, Península Ibérica.
}

Key words. Genista, Leguminosae, new combinations, Iberian Peninsula.

Willkomm (1877; 1893) incluyó bajo Genista lobelii DC. plantas de Castilla La Nueva (Sierra de Guadarrama, Serranía de Cuenca, Guadalajara), Cantabria (in montibus Potes, Aliva, Ler. et Lev. 1879) y SE de España (Sierra de Segura, Bourg., Alhama de Granada, Wbb., Sierra Tejeda, Bss., Wk., Sierra de María Wk., Fk.!, Sierra Sagra Bourg.! Velez Rubio (Rouy) Mugrón, Porta et Rigo, 1890, forma cinerea). Tras la ravisión de las genistas para Flora Iberica se llega a la conclusión de que las plantas de Cantabria pertenecen a Genista legionensis (Pau) M. Laínz, las de Castilla La Nueva y parte de las del SE de España a Genista pumila (Debeaux \& É. Rev. ex Hervier) Vierh., y las del resto del SE de España a Genista longipes Pau.

Genista legionensis (Pau) M. Laínz in Bol. Inst. Estud. Asturianos, Supl. Ci. 10: 194 (1964)

Genista aspalathoides var. legionensis $\mathrm{Pau}$ in Cavanillesia 1: 60 (1928) [basión.]

Genista hystrix subsp. legionensis (Pau) Gibbs in Notes Roy. Bot. Gard. Edinburgh 27: 57 (1966)

Genista polyanthos subsp. legionensis (Pau) M. Laínz, Contr. Fl. Asturias: 44 (1982)

Ind. loc.: "Rondiella, en los Picos de Europa, Lacaita (19,VII,1927); Oseja de Sajambre (Pau, 17, VII)"

Tipo: "Caroli Pau herbarium hispanicum
[Impreso] / Genista aspalathoides Link V.) legionensis Pau in lit. / La Rondiella: Picos de Europa / Legit Lacaita 19.VII-1927" [m. Pau]. Lectotypus: MA 58991, ejemplar con flores montado a la derecha del pliego. Este ejemplar fue propuesto por P. E. Gibbs (1966) como holótipo.

Tipificación: En el pliego (MA 58991) del lectótipo se encuentra una rama en fruto de Genista legionensis montada con una etiqueta de Carlos Pau que indica "Oseja de Sajambre / 17.VII.1927 / legi /C. Pau/var. legionensis Pau / = G. obelii Leresche et Levier". Esta planta forma también parte de la colección tipo y tiene órganos estipulares con espinas de hasta 1,3 $\mathrm{mm}$. El Lectótipo es la única planta, de c. $1,5 \mathrm{~cm}$, que se conserva de la recolección de Rondiella y tiene órganos estipulares con espinas de 0,7-0,8 mm, los cálices son viloso-seríceos con el tubo glabrescente y los estigmas son terminales y capitado-crestados.

Material estudiado: ESPAÑA: LEÓN: Entre Isoba y Puebla de Lillo, 1320 m, 29-IX-1973, E. Valdés \& G. López, MA 472128. Maraña, Pico del Escavar del Acebo, 8-VII-1980, sin recolector, MA 502808. Oseja de Sajambre, 17-VII-1927, Pau, MA 388671 (Sintipo de G. legionensis); idem, 12-VI1982, J. Andrés \& F. Llamas, MA 489152; SEV 99270. Posada de Valderón, 1500 m, 15-VIII-1967. Borja, MA 185286. OVIEDO: Covadonga, Lago de Covadonga, 5-VII-1953, Malato-Beliz, MA 280173; idem, lago de la Ercina, 14-VII-1952, E. Guinea, MA 336627. Puerto de Tarma, 7-VIII-1963, Galiano, SEV 1927. Sierra de Covadonga, 1150-1200 m, 1VIII-1960, Dresser, E. PALENCIA: Cervera de Pisuerga, Pico de las Cruces, 1360 m, 29-V-1990,

Este trabajo ha sido realizado gracias a los proyectos de la DGICYT (PB91-0070-C03-03) Flora Iberica IV y (PB96-1352) Flora Iberica V. 
Alejandre, MA 493829; idem, Monte Caderano, 1200 m, 5-VI-1990, Alejandre, MA 493828. Dehesa de Montejo, $1100 \mathrm{~m}, 4-\mathrm{VI}-1990$, Alejandre, MA 493761; idem, 1100-1180 m, 4-VI-1990, Alejandre, MA 493775; idem, Monte Mariserrana, 1200-2300 m, 4-VI-1990, Alejandre, MA 493776. SANTANDER: Altos de Aliva, 11-VII-1981, E. Fuertes, MA 394914; idem, 14-VIII-1980, Rico, MA 261877. Espinama, espolones calizos, $1300 \mathrm{~m}$, 2-VII-1983, Rivas Martínez, Cantó \& Sánchez Mata, MA 443407; SEV 155282. Espinama, Invernales de Igüedri, 1300 m, 2-VII-1983, Rivas Martínez, Cantó \& Sánchez Mata, MA 506013. Espinama, Refugio de Aliva, 1450 m, 19-VIII-1976, S. Catroviejo \& E. Valdés-Bermejo, MA 336061; idem, 20-VIII-1969, Sañudo, MA; idem, 7-VIII-1971, Talavera, Silvestre \& Valdés, SEV 15830; idem, subida a Aliva, El Collado, 1020-1600 m, 6-VII-1978, S. Catroviejo, G. López \& Valdés-Bermejo, MA336119. Fuente De, 2300m, 4-VII-1984, Izuzquiza \& al., MA 408267; idem, Cuetos de San Juan Toribios, 18001900 m, 5-VII-1978, Harrold \& McBeath, E. Fuente Deva, 1962-1963, Borja \& Rivas Martinez, MA 178510. Macizo de Andará, 16-V-1984, E. Fuertes, MA 388671. Peñas Viejas, 1800 m, 21-VIII-1952, Dresser, E; idem, 7-VIII-1971, Silvestre, Talavera \& Valdés, SEV 15830; idem, La Revoltona, 19621963, Borja \& Rivas-Martínez, MA 188891. Picos de Europa Occidental, Sierra Cebollera, $1200 \mathrm{~m}$, 25-VII-1928, Cuatrecasas, MA 167906. Picos de Europa, 16-VI-1954, Casaseca, MA 178501. Picos de Europa, Refugio Veronica, 2050 m, 8-VIII-1971, Boquet, Borel \& Reist, MA 336046; idem, El Cable, 1850 m, 11-VII-1984, M. Luceño, MA 347923. Potes, Valle de la Hermida, 200 m, 29-VIII-1969, Sañudo $\mathrm{n}^{\circ}$ G6, MA 293304; idem, 20-V-1970, Sañudo G6, MA 261867. VIZCAYA: Entre Abanto y Ciervana, 1800 m, 21-V-1989, Uribe Echevarria y Urrutia, MA 486029.

Observaciones: Genista legionensis se diferencia de todas las especies de la sección Erinacoides Spach de la Península Ibérica por tener el estigma terminal, capitado-crestado, parecido al de G. aspalathoides Lam. del NW de Argelia, Túnez y Sicilia. El resto de las especies tienen el estigma subterminal, elíptico e introrso. Esta especie es parecida en el porte a $G$. longipes Pau, de la que se diferencia por sus órganos estipulares espinosos y estandartes glabrescentes, parecidos a los de $G$. hystrix Lange, por cuyo motivo Gibbs (1966) la incluyó como subespecie dentro de la especie langeana. El estudio cariológico realizado por Sañudo $(1971 ; 1973)$ nos revela que $G$. legionensis tiene $\mathrm{n}=20, G$. histrix Lange y $G$. polyanthos $\mathrm{R}$. Roem. ex Willk. n $=12$, y $G$. longipes Pau y $G$. pumila (Debeaux \& É. Rev. ex Hervier) Vierh. $\mathrm{n}=$ 9 y $\mathrm{n}=18$ en ambas especies.

Lambinon repartió una centuria (Fasc. 19, 1980, 1981, 1983; n $\left.{ }^{\circ} 10343\right)$ de plantas supuestamente recolectadas por Segura-Zubizarreta en Pico Cabañas (Sierra de Cazorla, Jaén) bajo el nombre de G. lobelii subsp. longipes (Pau) Heywood, taxon muy frecuente en la Sierra de Cazorla, que sin lugar a dudas es $G$. legionensis (Pau) Laínz. Se ha visto el verdadero material recolectado en Pico Cabañas que muy amablemente nos envió Segura Zubizarreta, que sí es efectivamente $G$. longipes Pau. Sólo un cambio de etiquetas y el parecido aparente entre las dos especies podría explicar esta confusión de Lambinon

Genista pumila (Debeaux \& É. Rever. ex Hervier) Vierh. in Verh. Zool.-Bot. Ges. Wien 69: 181 (1919)

Genista baetica var. pumila Debeaux \& É. Rever. ex Hervier in Bull. Acad. Int. Géogr. Bot. 15: 65 (1905) [basión.]

Genista baetica subsp. pumila (Debeaux \& É. Rever. ex Hervier) Fern. Casas in Fontqueria 7: 17 (1985)

Genista versicolor subsp. pumila (Debeaux \& É. Rever. ex Hervier) Fern. Casas in Fontqueria 8: 31 (1985)

Ind. loc.: "E. Reverchon, in litt. 1901, et exsicc., nº 1263 (1901). Hab. Sierra de Cazorla, lieux arides, rare, $1600 \mathrm{~m}$ "

Tipo: "Elisée Reverchon - Plantes D'Espagne - 1901 / Province de Jaen / n 1263 / Genista baetica Spach / Forma pumila E. Reverchon / Sierra de Cazorla, Lieux arides, sur le calcaire, 1600 mètres. / rare. / Mai”. P. E. Gibbs (1966) indicó como holótipo el material de la centuria depositada en el herbario del Museo de Historia Nacional de Viena (W). Nosotros hemos visto un pliego de la centuria depositado en el Museo Británico de Historia Natural Londres (BM).

Tipificación: En el pliego del BM antes mencionado existe 2 trozos de plantas en flor con 2 etiquetas: una de revisión de P. E. Gibbs de 1962 y otra de Lectotipificación, no publicada, realizada 
por P. M. Uribe-Echebarria y P. Urrutia, hecha en 1990. El material tipo contenido en el pliego, formado por 2 fragmentos, tiene órganos estipulares truncados, bi o tridentados y las flores, de c. $11 \mathrm{~mm}$, tienen cálices de c. 3,5 mm y pedicelos de c. $3 \mathrm{~mm}$. Uribe-Echebarría \& Urrutia (1988), tras haber visto fotocopia del pliego del BM antes mencionado y visitado la Sierra de Cazorla, llegan a la conclusión de que $G$. pumila es $G$. lobelii subsp. longipes, opinión que no compartimos.

Variabilidad: Esta especie, endémica de los suelos calizos o margo-calizos de la mitad oriental de España se diferencia del resto de las especies de la sección Erinacoides por sus órganos estipulares truncados, bidentados, tridentados o redondeados en el ápice y sus tallos florales robustos.

En los matorrales xerofíticos montanos, sobre calizas, yesos o margas del SE de España, las plantas son de hasta $60 \mathrm{~cm}$, los órganos estipulares de los tallos viejos son frecuentemente muy verrugosos, bidentado o tridentado en el ápice y las flores, de 89,6(12) mm, tienen ovarios con 3-4 rudimentos seminales. Estas plantas se identifican con G. pumila (Debeaux \& É. Rev. ex Hervier) Vierh. s.s. (= G. mugronensis Vierh.).

En las parameras calcáreas del centro de España las plantas son generalmente más pequeñas (de hasta $20 \mathrm{~cm}$ ), los órganos estipulares de los tallos viejos son normalmente lisos, truncados 0 redondeados en el ápice y las flores, de (8)9-12 mm, tienen con frecuencia mayor número de rudimentos seminales. Estas poblaciones se identifican con $G$. rigidissima Vierh.

Estas diferencias nos parecen escasas y responden más bien a tendencias de variación de la especie. El porte grande o pequeño que presentan este grupo de papilionaceas suele ser una respuesta a condiciones climáticas y edáficas más benignas o severas respectivamente, y como indica Pau (1904) tiene escaso valor taxonómico. Plantas de $G$. rigidissima cultivadas por E. Fernández Galiano en el Jardín Botánico de Madrid procedente de Almadrones (Guadalajara) que en el campo no sobrepasan los $10 \mathrm{~cm}$, en cultivo superan $\operatorname{los} 40 \mathrm{~cm}$, cuyos testigos se encuentra en el herbario de Sevilla (SEV).

A pesar de que las diferencias morfológicas entre estos dos tipos de poblaciones son escasas, los estudios cariológicos realizados por Sañudo (l.c.) nos revelan que las poblaciones del sureste español son diploides $(\mathrm{n}=9 ; 2 \mathrm{n}=18)$ y las de los páramos castellano-aragoneses tetraploides $(n=18 ; 2 n=$ 36). Todo ello hace que consideremos a estos dos tipos de poblaciones con categoría de subespecies, que nos obliga a reajustar la nomenclatura.

\section{Genista pumila subsp. pumila}

Genista lobelii var. pumila Degen \& Hervier in Bull. Acad. Inst. Géogr. Bot. 17: 34 (1907) non Genista baetica var. pumila Debeaux \& É. Rev. ex Hervier

Genista mugronensis Vierh. in Verh. Zool.Bot. Ges. Wien 69: 180 (1919)

Genista pumila var. mugronensis (Vierh.) Heywood in Collect. Bot. (Barcelona) 5: 521 (1958)

Genista pumila subsp. mugronensis (Vierh.) Rivas Mart. in Publ. Inst. Biol. Aplicada 42: 119 (1967)

Material estudiado: ESPAÑA: ALBACETE: Almansa, 8-IX-1969, Sañudo, n ${ }^{\circ}$ 2, MA 293299; idem, Alto de Jódar, 27-V-1984, C. Obón, MA 312553. Chinchilla, 24-IV-1924, Pau, MA 58974. Mugrón de Almansa, 1-VI-1073, Borja, MA 505894. Sierra del Mugrón, 800 m, 6-IV-1976, M. Costa, MA207753. ALMERÍA: Sierra de Topares, IV-1962, Borja, MA 178526. Sierra de Gádor, 5-VI-1979, J. Varo, GDA 5899. Sierra María, Barranco de Mereva, $1500 \mathrm{~m}, 30-\mathrm{VI}-1978$, G. Blanca \& Cueto, Herb. Almería, SEV 136218; idem, cerca de los Cuernos, 1660 m, 8-VI-1988, Cueto, Herb. Almería, SEV 236232, SEV 136233; idem, cerca de la Loma de la Casa Blanca. $1100 \mathrm{~m}, 27-\mathrm{V}-1988$, G. Blanca \& M. Cueto, Herb. Almería, SEV 136230, SEV 136231; idem, Velez Rubio, El Maimón, 25-V-1988, G. Blanca \& M. Cueto, Herb. Almería, SEV 136228; idem, Cerro del Pontón, 22-VII-1987, M. Cueto, Herb. Almería, SEV 136217. ALICANTE: Entre Villar y El Vonete, 26-IV-1979, Asensi, MGC 5412. Villena, Sierra de Salinas, 29-V-1954, Rigual, MA 372981. CIUDAD REAL: Embalse de Peñarroya, 800 m, 15-V-1986, Lousac, MA 347910. Entre Ruidera y la Osa de Montiel, VII-1965, Borja \& Ladero, MGC 6136. Entre Ruidera y Peñarroya, 900 m, VII-1980, Peinado, MA 403931. Río Tovar, 4-V-1933, González Albo, MA 58965. GRANADA: Cortijo del Parador Pedro Martínez, 1000 m, 23-VI1983, Fernández Casas, MA 345435. Dehesa de Guadix, 900 m, 10-VI-1971, E. Sánchez \& Fernández Casas, MA 413479. Entre Almidar y 
Baños de Alicum, 750 m, 30-IV-1988, UribeEchebarría \& al., MA 454410. Hernán Valle, 5-VI1974, Fernández Casas, MA 329176. JAEN: Barranco del río Segura, $1500 \mathrm{~m}$, VI-1906, E. Reverchon in Reverchon PI. D'Espagne 1906: $\mathrm{n}^{\circ}$ 1263 E; MA 58973. Sierra de Cazorla, 1600 m, V1901, E. Reverchon in Reverchon Pl. D'Espagne 1901: $\mathrm{n}^{\circ}$ 1263, MB (tipo de Genista pumila). TOLEDO: Trevenque, El Valle, 18-V-1982, S. Laorga, SEV 90925. MADRID: Aranjez, 14-VII1934, sin recolector, MA 162184. MURCIA: Jumilla, El Garche, 1320 m, 30-IV-1977, Muñoz Garmendia \& al., MA 409566; idem, 1250 m, 4-VI1981, J. Molero, MA 261880. Sierra de Culebrilla, Cerro Sastre, sin fecha, sin recolector, SEV s/n. VALENCIA: Ayora, 950 m, 23-VIII-1987, UribeEchebarría \& al., MA 454411 . Benecas (Requena), 900 m, VI-1987, E. García, MA 544410. Pico del Caroch, IV-1982, Regueio, MA 415761.

Observaciones: Degen \& Hervier (1907) publicaron $G$. lobelii var. pumila basada en una planta recolectada por Reverchon en la Sierra del Pozo (Jaén) en 1905 (exscc. n 1263) por lo que debe ser considerada como una variedad distinta de G. baetica var. pumila publicada por Hervier en 1905 y que es el basiónimo de G. pumila.

Genista pumila subsp. rigidissima (Vierh.) Talavera \& Ll. Sáez com. nov. en este trabajo

Genista rigidissima Vierh. in Verh. Zool.-Bot. Ges. Wien 69: 181 (1919) [basión.]

Genista mugronensis subsp. rigidissima (Vierh.)

Fern. Casas in Fontqueria 7: 18 (1985)

Ind. loc.: "Hispania centralis. Prope Villar de Corbeta. Guadalajara. Herb. Torre de Pando als G. Iobelii (UV)"

Tipo: No estudiado

Material estudiado: ESPAÑA: CUENCA: De Campillo de Montebuy a Enguidanos, 2-V-1976, G. López \& al., MA 347944. Ensanche de Buenache, 30-X-1931, Huguet del villar, MA 159096. Maraña, Pico del Escavar del Acebo, 8-VII-1980, sin recolector, MA 502808. Pinar de Beteta, 8-VII1932, A. Caballero, MA 58963. Serranía de Cuenca, 1962, Borja, MA 188878; idem, Ciudad Encantada, 20-VI-1969, Rivas Goday, SEV 27690; idem, 15-V1977, Muñoz Garmendia, MA 208735. Solán de Cabras, 13-VI-1942, A. Caballero, MA 58964. GUADAlAJARA: Alcolea del Pinar, 1300 m, $7-$ VI-1976, Rivas Martinez, SEV 44044; idem, 13-V-
1962, Fernández Galiano, SEV S/N. Almadrones, 12-V-1962, E. Fernández Galiano, SEV 15374; idem, cultivada en el Jardín Botánico de Madrid, IX-1965, E. Fernández Galiano, SEV 114708, SEV 11465. Maranchón, 1250 m, 18-VI-1982, Rico \& Sánchez, SEV 92927; idem, alrededores del Puerto, 3-VII-1984, C. Soriano \& L. Sánchez, MGC 14677; SEV 111065. Sacedón, 6-IV-1969, Sañudo n ${ }^{\circ}$ G 6, MA 261920. Tamajón, 12-V-1944, Rivas Goday \& Monasterio, MGC 6150; SEV 30784; MA 261921. TERUEL: Almagro, Blancas, 1894, Pau, MA 397533. Entre Pozondón y Orihuela del Tremedal, 18-VI-1907, Pau, MA 397529. Monreal del Campo, VII-1894, Benedicto, MA 58958. Orihuela del Tremedal, VI-1907, Pau, MA 58963. ZARAGOZA: Entre Cetrina y Cimbacla, 12-V-1976, J. Izco \& S. Cirujano, MA 336655. Tabuenca, Sierra de la Nava Alta, 990 m, 10-VI-1989, Zúñiga \& Alejendre, MA484596. SORIA: Arbujuelo, 1200 m, 4-VI-1977, Segura Zubizarreta, SEV 55700. Arcos de Jalón, 4VI-1034, L. Ceballos, MA 58961. Burgo de Osma, 12-VII-1969, Sañudo n ${ }^{\circ}$ G3, MA 261920; idem, 880 m, 17-VI-1997, M. Carrasco, S. Castroviejo, B. Casaseca \& S. Talavera, SEV 155053. Sierra de Moedo, 9-VI-1935, C. Vicioso, MA 58960. Ucero La Galiana, 9-VI-1933, L. Ceballos, MA 58962. Vililla de la Sierra, 1100, 27-VI-1978, SeguraZubizarreta, SEV 75946. VALENCIA: Monte Palomera, 20-VI-1906, Pau, MA 58957.

Localidad no encontrada: Castilla. Sierra Minista, 26-V-1896, M. Gangoder, E.

Genista longipes Pau in Bol. Soc. Aragonesa Ci. Nat. 3: 282 (1904)

Genista lobelii subsp. longipes (Pau) Heywood, Collect. Bot. (Barcelona) 5: 519 (1958)

Ind. loc.: "Abunda en la cumbre junto al mollonet, de donde proceden los ejemplares traídos" [Sierra de Aitana, Alicante]

Tipo: No estudiado.

Ecología y distribución: En matorrales almohadillados de alta montaña, con suelo calcáreo o dolomítico, entre 1300-2300 m, del S y SE de España [Sierra Aitana (Alicante), Sierra Seca (Murcia), Sierra de Villafuerte (Murcia), Sierra Espuña (Murcia), Macizo de Cazorla-Segura (Jaén), Sierra de Mágina (Jaén), La Sagra (Granada), Sierra Nevada (Granada), Sierra de Filabres (Almería), Sierra María (Almería), Sierra Tejeda-Almijara (Granada-Málaga) y Sierra de las Nieves (Málaga). 
Variabilidad: Esta especie se diferencia del resto de las especies de la sección Erinacoides por sus tallos fértiles delgados y por sus fruto fusiformes y falcados. Heywood (1958) y Uribe-Echebarria \& Urrutia (1988) incluyeron este taxon como subespecie de $G$. lobelii DC. pero la planta del sur de Francia presenta los frutos oblongoideos, como el resto de las especies de la sección Erinacoides, y está emparentada al menos fenéticamente con $G$. pumila.

Las poblaciones más orientales del área de distibución de la especie, que habitan en las collados calcáreos de Alicante, Murcia, Sierra de Segura, La Sagra, Sierra María y Sierra de Filabres están formadas por individuos muy compactos, casi afilos durante la floración, tallos adultos generalmente con 8 costillas que ocultan o casi ocultan los valles intercostales, órganos estipulares de los tallos viejos frecuentemente cónicos y cálices, de 3-4(5) mm, con los dientes del labio inferior de menos de $1 \mathrm{~mm}$. Estas plantas se han identificado con $G$. longipes Pau s.s. descrita de la Sierra de Aitana.

Las poblaciones más occidentales del área, que habitan en los suelos dolomíticos de la Sierra de Mágina, Sierra de Cazorla, Sierra Nevada, Sierra Tejeda, Sierra de Almijara y Sierra de la Nieves están formadas por individuos menos compactos, frecuentemente con numerosas hojas durante la antesis, tallos adultos generalmente con 10 costillas que dejan ver los valles intercostales, órganos estipulares de los tallos viejos frecuentemente truncados y cálices, de 4-6 mm, con los dientes del labio inferior de 1-1,6 mm. Estas poblaciones se identifican con $G$. tejedensis (Porta \& Rigo ex Hervier) Vicioso p. p.

Estas diferencias parecen suficientes como para considerar a ambos tipos de poblaciones como subespecies: G. longipes subsp. longipes para designar a las poblaciones calcícolas más orientales y G. longipes subsp. viciosoi que se describe como nueva para las dolomíticas occidentales, en honor al ilustre botánico que tanto trabajó en las genisteas de España en los años 50.

\section{Genista longipes subsp. longipes}

Genista lobelii var. tejedensis Porta \& Rigo ex Hervier in Bull. Acad. Int. Géogr. Bot. 15: 65 (1905) p.p. (excl. loc. Sierra Tejeda)

Genista tejedensis (Porta \& Rigo ex Hervier) C. Vicioso in Bol. Inst. Forest. Invest. Exp. 67:
42 (1953) p.p.

Observaciones: Hervier (1905: 65) en la publicación de $G$. lobelii var. tejedensis indica materiales de 3 localidades ["Sierras de Maimon, del Cuarto et de Castril"] que Reverchon había repartido como exsiccatas $\mathrm{n}^{\circ} 1127$ bajo G. lobelii var. longepedunculata y de otras dos ["Sierra de Tejeda (1879) et de la Sierra María (1890)"] de sendas exsiccatas $n^{\circ} 546$ de Porta y Rigo que fueron repartidas con el nombre de G. lobelii var. tejedensis. De todo este material citado, el más antiguo es el de Sierra Tejeda recolectado por Porta y Rigo en 1879, y posiblemente por ello Hervier usa el nombre que estos autores habían dado a la planta de su exsiccata. La única diagnosis que aparece en la descripción de Hervier de G. lobelii var. tejedensis es la siguiente: "C'est une forme ou variété assez remarquable qui diffère du type par ses pédicelles deux fois longs (parfois même plus longs) que le calice; le type (D.C. fl. Fr. IV, p. 500) a les fleurs portées sur des pédicelles courts". En base a la descripción, el material tipo debe ser elegido de entre las plantas con pedicelos dos veces más largos o más que el cáliz, carácter que le conviene a la mayoría de las plantas de las localidades del protólogo pero no a las de la exsiccata de Porta y Rigo de Sierra Tejeda. El pliego de Sierra Tejeda de estos autores que se conserva en el Jardín Botánico de Edinburgo (E) lo compone 2 plantas con pedicelos de 4-6 $\mathrm{mm}$, casi del tamaño del cáliz (" $n^{\circ} 546$ Herb. de Porta et Rigo Itinere Hisp. 1879 / Genista baetica / b tejedensis nov. / Regnum granatensis clivibus glareis petrosis I in Sierra Tejeda parte septentr. 15-1700 m sol. calcar. / 23 Juni" [m. Porta]). El material de la Sierra de Maimón ("E. Reverchon Pl. Esp. 1899 / Prov. Almería / no 1127 Genista lobelii DC. / Sierra de Maimon, lieux / arides et rocheux sur le calcaire / 1700 m / var. longepedunculata Debeaux" [m. E. Reverchon]) que se conserva en el herbario de Edinburgo está formado por cinco trozos de plantas con pedicelos largos y glabrescentes la mayoría de ellos más de 2 veces más largos que el cáliz. Este material de Reverchon de la Sierra de Maimón, que se ajusta perfectamente a la descripción original se elige como lectótipo de Genista lobelii var. tejedensis (LECTOTYPUS: se elige como lectópipo en este trabajo el ejemplar superior del pliego).

Material estudiado: ESPAÑA: ALICANTE: Sierra Aitana, VI-1944, Borja, MA 186996; idem, 4-VII-1949, Rivas Goday \& al., MA 261883; idem, 
Compides, 1300 m, 9-VII-1958, Rigual, MA 272967; idem, 19-IV-1936, F. Cámara, MA 58983; idem, 1500 m, 15-VI-1923, Font Quer, Fl. Iber. Selecta Cent. III, 1935, MA 58982; idem, cumbre de la Sierra, sin fecha, E. F. Galiano, SEV 1930; idem, 4VII-1949, E. F. Galiano, SEV 1929. ALMERÍA: Cerca de Vélez Blanco, 1300 m, 30-VII-1987, M. Cueto, SEV 136234. Sierra de Filabres, Calar del Gallinero, 1900 m, VI-1989, G. Nieto, MA 468914; idem, Bacares, 12-VI-1929, Gros, MA 58975; idem, 2000 m, 10-VI-1994, A. Pallares, MA 542212. Sierra María, cerca de la buitrera, $1600 \mathrm{~m}, 15-\mathrm{V}-1987, M$. Cueto, Herb. Almería, SEV 136235, SEV 136236. Sierra María, Pico Argerúm, 1700 m, 2-VI-1987, G. Blanca \& M. Cueto, Herb. Almería, SEV 136237. Sierra María, Pico Enjambre, 1770 m, 3-VI-1987, G. Blanca \& M. Cueto, Herb. Almería, SEV 136238. Sierra María, Velez Blanco, El Maimón, 1150-1500 m, 17-VII-1980, G. López \& R. Morales, MA 343243, SEV 148392; idem, Pico Cabezo, 1800 m, 1-VII-1987, G. Blanca \& Cueto, Herb. Almería, SEV 136242; idem, 1700 m, VI-1899, E. Reverchon PI. D’Espagne 1899: n 1127, E (Lectótipo de G. lobelii var. tejedensis); idem, VI, 1899, E. Reverchon in Dörfler Herb. Normale $n^{\circ} 4621$, E (Isolectótipo de G. lobelii var. tejedensis); idem, $1750 \mathrm{~m}, 2-\mathrm{V}$ 1970, Fernández Casas, MA 410640; idem, Vélez Blanco, El Maimón, C. N., 1150-1610 m, G. Blanca \& al., Herb. Almería, SEV 136184; idem, Vélez Rubio, El Maimón, 25-V-1988, G. Blanca \& M. Cueto, Herb. Almería, SEV 136127; idem, Cima de Sierra María, 1720 m, 21 -VI-1988, M. Cueto, Herb. Almería, SEV 136241; idem, Cerca de Portachico de Sierra María, 21-VI-1988, M. Cueto, Herb. Almería, SEV 136240; idem, Barranco Molina, Vertiente sur, $1900 \mathrm{~m}, 4-\mathrm{VI}-1987$, G. Blanca \& Cueto, Herb. Almería, SEV 136239. Vélez Rubio, Sierra de Maimón, VII-1960, Rivas Martínez, SEV 32394. GRANADA: Sierra de la Cabrilla, $1800 \mathrm{~m}$, VI-1905, E. Reverchon in Dörfler Herb. Normale $\mathrm{n}^{\circ}$ 4621, E, MA 177222, MA 58979; idem, VI-1905, E. Reverchon, 1905: $n^{\circ} 1127$, E, MA 58980, MA 58981, MA 58979. Sierra de la Sagra, 6-VI-1851, E. Bourgeau PI. Esp. 1851: n 1133, E; idem, 1850$2300 \mathrm{~m}, 24-\mathrm{VI}-1988$, Talavera \& Valdés, SEV 155052. Sierra del Castril, 8-VII-1992, Passera, GDA 37614. JAEN: Sierra de Segura, El Yelmo, 1700 m, 21 -VI-1979, S. Pajarón, MA 580801; idem, 11-VI-1975, Fernández Casas \& Fernández Piqueras, MA 261879; idem, Cortijos Nuevos, 1900 m, 30-VI-1988, Aparicio, García \& Silvestre, MA 486309, SEV 155050; idem, 1500-1800 m, 20-VII1977, S. Castroviejo \& E. Valdés, MA 3923903; idem, 1800 m, 22-IV-1995, Heywood, MA 188736; idem, El Calarejo Grande, 1600 m, 1-VII-1955, Heywood, MA 188735. MURCIA: Sierra de Villafuerte, Collado Grande, al W del Puntal de la Vieja, 1450 m, 14-V-1992, Uribe-Echebrría, MA 537994. Sierra Espuña, 23-VI-1947, C. Vicioso, MA 151078; idem, 1450 m, 25-IV-1970, Sañudo n ${ }^{\circ}$ G1, MA 261878; idem, 4-V-1964, E. Fernández Galiano, SEV 155051. Sierra Seca, Moratalla, Pico Revolcadores, 1800 m, 22-V-1982, C. Selma, MA 541606; idem, Cañada de la Cruz, 11-X-1964, E. F. Galiano \& J. Novo, SEV 9625

Genista longipes subsp. viciosoi Talavera \& Cabezudo subsp. nov. en este trabajo

Typus: Málaga. Yunquera. P. N. Sierra de la Nieves. Entre la Cañada de los Hornillos y el Peñón de los Enamorados. 30SUF2265. 1350-1750 m, 18VI-1998, leg. Cabezudo, Pérez Latorre \& al. HOLOTYPUS: SEV 154277; isotypus MA, MGC 46812.

Humilis ramosissima caespitosa foliosa in anthesis cum ramis sultato-striatis generaliter 10 costatis, pungentis; floribus solitariis pedicelatis, cun pedicelis 2-7(11) mm longis generaliter sericeis, calycis 4-6 mm longis cum laciniis inferioribus 1$1,7 \mathrm{~mm}$ longis.

Material estudiado: ESPAÑA: GRANADA: Alhama de Granada, Sierra Tejeda, 1-VII-1993, Cabezudo \& als, MGC 36223. Sierra de Almijara, Otivar, subida al Navachica, cara W, 11-VII-1991, B. Cabezudo \& al., SEV 141232. Sierra Nevada, Quentar, Cerro Obscuro, barrancada umbría, 19VIII-1983, Molero Mesa \& Pérez Raya, GDAF 17899. Sierra Nevada, sin fecha, E. Fernández Galiano, SEV 1911. Sierra Tejeda septentrional. 1500-1700 m, 30-V-1879, Porta \& Rigo iter Hisp. 1879: n $^{\circ}$ 546, E. JAÉN: Peal de Becerro, Peña Juana, 1820 m, 7-VII-1976, Muñoz Garmendia \& C. Soriano, MA 481592. Quesada. Entre Puerto Llano y la cabecera del Barranco del Escalón, 1750 m, 26VI-1975, González Rebollar, MA 481267. Sierra de Cazorla, Loma de los Castellones, 1600 m, 22-VI1975, González Revollar \& C. Soriano, MA 457251; idem, Pico Cabañas, 20-VI-1978, Varo \& al., GDAC 5420; idem, 1900m, 14-VII-1980, C. Soriano, MA 457252; idem, 23-VII-1974, Fernández Casas, MA 
261881. Sierra Mágina, entre Cambil y Huelma, 1500 m, 15-VI-1982, Molero Mesa \& al., MA 393914. MÁLAGA: Sierra de la Nieves, Yunquera, 1350-1750 m, 18-VI-1998, Cabezudo, Pérez Latorre, Gil, Navas \& Navas, SEV 154277, MGC 46812, MA s/n (tipo de Genista longipes subsp. viciosoi); idem, 15-VII-1998, Cabezudo, Pérez Latorre, Gil, Navas \& Navas SEV, MGC 46812. Sierra Tejeda, VI-1837, Boissier, E; idem, Cómpeta, 2-VI-1931, C. Vicioso, MA 162211; idem, 11-VI1919, Font Quer, MA 58999; idem, Casa de las Nieves, 27-V-1982, Nieto, MGC 20022; idem, La Maroma,1-V-1980, Nieto, MGC 18999.

\section{BIBLIOGRAFÍA}

GIBBS, P. E. - 1966- A Revision of the Genus Genista L. Notes Roy Bot. Gard. Edinburgh 27: 11-99

HER VIER, J. -1905- Excursions botaniques de M. Elissée Reverchon. Bull. Acad. Int. Géogr. Bot. 15: 65

HER VIER, J. -1907- Excursions botaniques de M. Elissée Reverchon. Bull. Acad. Int. Géogr. Bot. 17: 34

HEYWOOD, V. H.. - 1958- The taxonomy and distribution of Genista lobelii DC. and allied Species. Collect. Bot. (Barcelona) 5: 515-525

PAU, C. -1904- Plantas de la Sierra de Aitana (Alicante). Bol. Soc. Aragonesa Ci. Nat. 3: 279288

SAÑUDO, A. -1971- Variabilidad cromosómica de las Genisteas de la Flora Española en relación con su ecología. I. Núm. y comportamiento de los cromosomas durante la meiosis. A. Secciones Erinacoides Spach, Scorpioides (L.) DC y Asterospartum Spach del Gén. Genista. Cuad. Ci. Biol. 1: 1-21

SAÑUDO, A. -1973- Variabilidad cromosómica de las Genisteas de la Flora Española en relación con su ecología. Apéndice. Cuad. Ci. Biol. 2(2):
123

URIBE-ECHEVARRÍA, P. M. y P. URRUTIA 1988- Apuntes para el conocimiento de la sección Erinacoides Spach del género Genista L. (Leguminosae). Est. Inst. Alavés Natur. 3: 209-224

VIERHAPPER, F. -1919- Beiträge zur kenntris der Flora Griechenlands; A. Anthophyta und Pteridophyta. Genista. Verh. Zool.-Bot. Ges. Wien 69: 157-185

WILLKOMM, M. in WILLKOMM, M. \& J. LANGE -1877- Prodromus Flora Hispanicae 3: 431. Stuttgartiae

WILLKOMM, M. -1893-Supplementum Prodromus Flora Hispanicae: 253. Stuttgartiae

Aceptado para su publicación en Noviembre de 1998

Dirección de los autores. Departamento de Biología Vegetal y Ecología, Universidad de Sevilla. Apdo 1095, E-41080 Sevilla 